\title{
Correction
}

\section{Correction: Shi et al., Shank Proteins Differentially Regulate Synaptic Transmission (eNeuro November/December 2017, 4(6) 0163-15.2017 1-12 https://doi.org/10.1523/ ENEURO.0163-15.2017)}

In the article "Shank Proteins Differentially Regulate Synaptic Transmission," by Rebecca Shi, Patrick Redman, Dipanwita Ghose, Yan Liu, Xiaobai Ren, Lei J. Ding, Mingna Liu, Kendrick J. Jones, and Weifeng Xu, which appeared as e0163-15.2017 in the November/December 2017 issue, 4(6), author Hongik Hwang was inadvertently omitted from the author list. The authors apologize for the oversight. The corrected author list is as follows: Rebecca Shi ${ }^{1,2,3}$, Patrick Redman ${ }^{1}$, Dipanwita Ghose ${ }^{1}$, Hongik Hwang ${ }^{1,4}$, Yan Liu ${ }^{1}$, Xiaobai Ren ${ }^{1}$, Lei J. Ding ${ }^{1,3}$, Mingna Liu ${ }^{1}$, Kendrick J. Jones ${ }^{1}$, and Weifeng Xu ${ }^{1,2}$. Their affiliations are as follows: ${ }^{1}$ Picower Institute for Learning and Memory, ${ }^{2}$ Department of Brain and Cognitive Sciences, ${ }^{3}$ Department of Biology, ${ }^{4}$ Department of Chemistry, Massachusetts Institute of Technology, Cambridge, MA 02139. The Author Contributions footnote should have read "Author contributions: P.R. and W. X. designed the experiments; R.S., P.R., H.H. and D.G., performed electrophysiology recordings; L.J.D., K.J., Y.L., X.R., and R.S. performed the biochemistry; P.R., M.L., R.S., H.H., D.G., and W.X. analyzed data; R.S. and W.T. wrote the paper." This correction does not affect the conclusions of the paper. The 4th author has been added in the online PDF version. 